CONCISE REPORT

\title{
Ankylosing enthesitis, dactylitis, and onychoperiostitis in male DBA/1 mice: a model of psoriatic arthritis
}

\author{
R J U Lories, P Matthys, K de Vlam, I Derese, F P Luyten
}

Ann Rheum Dis 2004;63:595-598. doi: 10.1136/ard.2003.013599

\begin{abstract}
Objectives: To further characterise spontaneous arthritis in aging male DBA/ 1 mice as a model of spondyloarthropathy and psoriatic arthritis with particular attention to signs of inflammation and nail involvement.

Materials and methods: Aging male DBA/1 mice from different litters were caged together (4-6 mice per cage) at the age of 12 weeks, checked twice a week for signs of arthritis, and killed at different times. Hind paws were dissected and processed for histology.

Results: Disease incidence varied between $50 \%$ and $100 \%$ in four different experiments. Besides clinical signs of arthritis, nail abnormalities were noticed. Pathological examination showed the occurrence of dactylitis characterised by diffuse neutrophil infiltration in 6 of 50 paws examined. Onychoperiostitis with progressive destruction of the nail bed and the underlying distal phalanx was seen in 5 of 50 paws examined.

Conclusions: Although dactylitis and onychoperiostitis are rare manifestations of the disease process, these data strongly suggest that spontaneous arthritis in aging male $\mathrm{DBA} / 1$ mice shares important features with human psoriatic arthritis. This model may therefore be an important tool to study links between stress, sex, inflammation, and new bone formation with particular relevance to human psoriatic arthritis.
\end{abstract}

$\mathrm{P}$ soriatic arthritis (PsA) is a common and destructive chronic inflammatory joint disease with a long term functional outcome that appears to be comparable to rheumatoid arthritis. ${ }^{1}$ Specific clinical signs of PsA include ankylosing enthesitis (AE) in the peripheral joints and dactylitis. ${ }^{2}$ Primary enthesitis is suggested to be the characteristic feature. ${ }^{3}$ Therefore PsA is part of the spondyloarthropathy (SpA) concept. ${ }^{4}$ Psoriatic nail lesions such as onycholysis, formation of subungual oil spots, and pitting nails are often encountered in patients with PsA. Onychopachydermo-periostitis (OPP) with periosteal cell proliferation and erosion of the distal phalanx is a less common yet pathognomic sign of PsA. ${ }^{56}$

Spontaneous arthritis in aging male DBA/l mice ( $\mathrm{SpAD}$ ) is characterised by AE in the peripheral joints of the hind paws. Its pathology is strongly reminiscent of human SpA and PsA in particular. ${ }^{7}$ Despite clinical signs suggesting important joint inflammation, ${ }^{8}$ the specific relevance of this model to human chronic arthritis has been debated, given the absence of inflammatory infiltrates in the synovium ${ }^{7}$ as well as the observation that both T cell receptor $\alpha \beta$ and $\gamma \delta$ knockout mice develop disease with similar incidence and severity as wildtype mice. ${ }^{7}$

We suggested that not all disease manifestations may have been described in this model. A hitherto unrecognised inflammatory reaction may precede signs of AE. Therefore, we examined mice at different ages with or without symptoms. We report that not only AE but also dactylitis and nail involvement in SpAD are strikingly similar to human SpA and, in particular, to PsA.

\section{MATERIALS AND METHODS \\ Animal experiments}

In four different experiments, 33 male DBA/1 mice from different litters were caged together at the age of 12 weeks (4-6 mice per cage) and checked twice weekly for clinical signs of arthritis. In the first three experiments animals were killed 7 (age 21 weeks, 10 mice) or 8 weeks (age 24 weeks, $2 \times 8$ mice) after the first symptoms in the cohort developed. In one experiment the animals were killed 4 weeks after the first signs of disease occurred (age 25 weeks, 7 mice). The local Ethical Committee for Animal Experiments approved the procedures.

\section{Pathology}

A complete microscopic analysis of forefeet from the hind paws was performed in 25 animals (experiments 2-4). Mice hind paws were dissected, fixed in formalin for 16 hours, decalcified using Decal (Serva, Heidelberg, Germany) for 72 hours, dehydrated, and embedded in paraffin. Sections were cut in a transversal plane allowing analysis of all interphalangeal and metatarsophalangeal joints. Sections were stained with haematoxylin and eosin.

\section{RESULTS}

In four different experiments, 33 mice were studied (table 1). Complete pathological analysis was performed in 25 mice (table 1). Disease incidence varied between 50 and 100\% in different experiments (table 1). Our clinical observations were largely similar to those previously reported. ${ }^{7}$ Joint swelling, joint stiffness as demonstrated by loss of grip function on the cage grid, and joint deformity were the major symptoms (table 1). We also noticed an abnormal position of the nail in affected animals suggesting specific involvement of the nail bed and/or distal phalanx. At the end of the observation period 26/66 paws (39\%) appeared normal. A score 1 (one toe affected ${ }^{7}$ was given to $10 / 66$ paws (15\%), a score 2 (more than one toe affected) was assigned to $6 / 66$ paws $(9 \%)$. Progressive disease as demonstrated by loss of grip function due to toe stiffness (score 3) was found in 11/66 ( $17 \%)$ paws. Ankle arthritis or persisting deformity of a toe (score 4 ) was seen in $13 / 66$ paws $(20 \%)$. In addition, nail involvement was noticed in $8 / 66(12 \%)$ paws.

Three different manifestations of the disease were recognised by microscopic analysis (table 1). Typical signs of AE characterised by endochondral bone formation were similar

Abbreviations: $A E$, ankylosing enthesitis; OPP, onycho-pachydermoperiostitis; PsA, psoriatic arthritis; SpA, spondyloarthropathy; SpAD, spontaneous arthritis in aging male DBA 1 mice 
Table 1 Clinical and pathological evaluation of spontaneous arthritis in aging male DBA/1 mice

\begin{tabular}{|c|c|c|c|c|c|c|}
\hline & \multicolumn{4}{|c|}{ Experiment } & \multirow[b]{2}{*}{ Total } & \multirow[b]{2}{*}{ Percentage } \\
\hline & 1 & 2 & 3 & 4 & & \\
\hline Observation time (weeks) & 4 & 7 & 8 & 10 & & \\
\hline Number of animals & 7 & 8 & 10 & 8 & & \\
\hline Disease incidence (\%) & 72 & 50 & 70 & 100 & & \\
\hline Normal paws & 5 & 10 & 7 & 4 & 26 & 39 \\
\hline Score 1: 1 toe affected & 3 & 2 & 3 & 2 & 10 & 15 \\
\hline Score 2: $>1$ toe affected & 0 & 2 & 3 & 1 & 6 & 9 \\
\hline Score 3: loss of grip function & 4 & 1 & 2 & 4 & 11 & 17 \\
\hline Score 4: persisting deformity or ankle arthritis & 2 & 1 & 5 & 5 & 13 & 20 \\
\hline Nail involvement & 3 & 2 & 0 & 3 & 8 & 12 \\
\hline Dactylitis* $^{*}$ & 2 & 2 & 2 & ND & 6 & 12 \\
\hline Enthesial proliferation* & 6 & 6 & 10 & ND & 22 & 46 \\
\hline Enthesial cartilage formation* & 5 & 5 & 9 & ND & 19 & 38 \\
\hline Enthesial bone formation* & 1 & 2 & 8 & ND & 11 & 22 \\
\hline Joint space bridging* & 1 & 2 & 8 & ND & 11 & 22 \\
\hline Onychoperiostitis* & 3 & 2 & 0 & ND & 5 & 10 \\
\hline
\end{tabular}
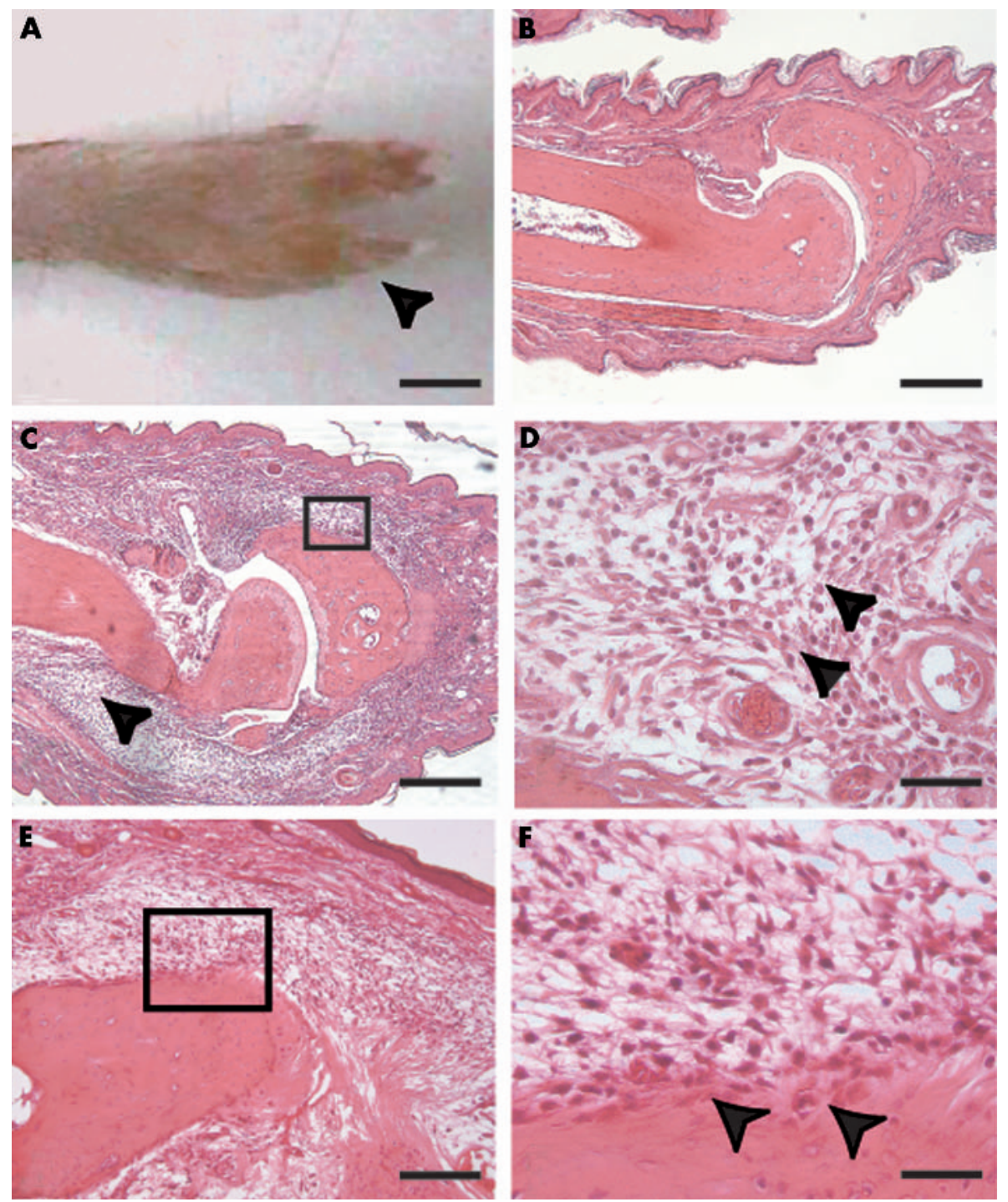

Figure 1 Dactylitis in spontaneous arthritis in aging male DBA/1 mice. (A) clinical signs of dactylitis (sausage digit) in the 4th toe (arrowhead); (B) microscopic image of a normal toe with a proximal interphalangeal joint; (C) microscopic image of dactylitis showing extensive subcutaneous oedema and tenosynovitis with disruption of muscle and tendon fibres (arrowhead); (D) microscopic detail showing neutrophils (arrowheads) in the subcutaneous tissue; $(E)$ inflammatory reaction adjacent to the enthesis; $(F)$ microscopic detail showing polynuclear and mononuclear cells (arrowheads) at the enthesis. (A) Bar $=2.5 \mathrm{~mm}$. Haematoxylin and eosin staining; bar $=400 \mu \mathrm{m}(\mathrm{B}, \mathrm{C}), 200 \mu \mathrm{m}$ (E), and $50 \mu \mathrm{m}$ (D-F). 

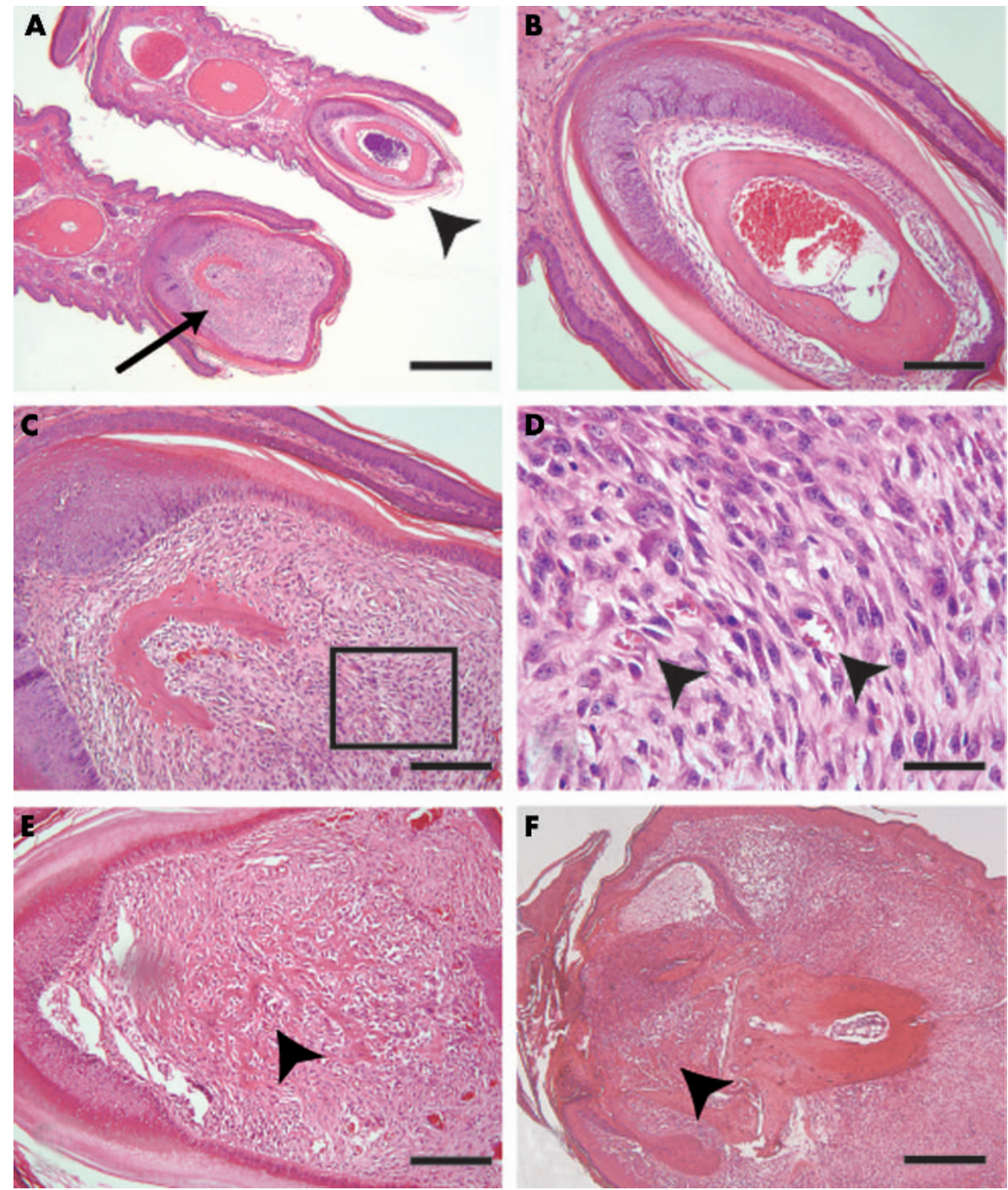

Figure 2 Onychoperiostitis in spontaneous arthritis in aging male DBA/1 mice. (A) Overview showing a normal toe and nail with the underlying distal phalanx (arrowhead) as well as a toe with an affected nail and distal phalanx periostitis (arrow); (B) detail of normal nail bed and distal phalanx ; $C$ and $E$ ) periostitis in diseased nails showing a hypercellular tissue $(C)$ and bone destructive, pannus-like tissue (E) invading the underlying distal phalanx (arrowhead); (D) detail from C showing spindle shaped cells and some small blood vessels (arrowheads); (F) late stage onychoperiostitis resulting in complete destruction of the nail bed and the distal phalanx (arrowhead). Haematoxylin and eosin staining; bar $=800 \mu \mathrm{m}(A), 400 \mu \mathrm{m}(\mathrm{F}), 200 \mu \mathrm{m}$ (B, $\mathrm{C}, \mathrm{E})$, and $50 \mu \mathrm{m}(\mathrm{D})$.

to those previously reported. ${ }^{7}$ AE occurs rapidly. Already after 4 weeks of clinical symptoms abnormal cartilage and bone formation were found in 5/14 and $1 / 14$ paws respectively (table 1). Eight weeks after clinical disease onset, cartilage formation was seen in 9/20 paws. Enthesial bone formation leading to joint space bridging was seen in $8 / 20$ paws at this time (table 1).

We also noticed dactylitis and onychoperiostitis. The unexpected presence of dactylitis was noticed in 6/50 paws (table 1, fig 1). In contrast with the normal toes (fig 1B), the "sausage" digit was characterised by extensive subcutaneous oedema and an acute inflammatory reaction involving numerous neutrophils (figs $\mathrm{IC}$ and $\mathrm{D}$ ). No specific signs of inflammatory infiltrates within the synovium were recognised. In contrast, tenosynovitis with perimuscular and tendon sheet oedema as well as cell infiltration were seen (fig IC, arrowhead). The overlying skin appeared normal. There were no signs of hyperkeratosis or dermal hyperplasia.

In two animals the acute inflammatory reaction apparently affected the enthesis itself, suggesting a link between the occurrence of dactylitis and AE (fig $1 E$ ). No inflammatory cells were found within the fibrocartilage, but neutrophils, some mononuclear cells, and fibroblast-like cells were found at the border of the enthesis in close contact with the underlying bone and fibrocartilage (fig lF).

In addition, sections through affected nails showed broadening of the nail base (fig 2A) and extensive cell proliferation above the distal phalanx, suggesting subonychal periosteal cell activation (fig 2C). Cells within this tissue mainly had a spindle shaped appearance and had prominent nucleoli (fig 2D). A few small blood vessels were found in the abnormal tissue (fig 2D). We found cells invading the bone, suggesting the presence of a periosteum derived, pannus-like tissue (fig 2E). In advanced stages the process of onychoperiostitis had led to the complete destruction of the distal phalanx and the overlying nail (fig $2 \mathrm{~F}$ ).

\section{DISCUSSION}

We have demonstrated AE, dactylitis, and onychoperiostitis in a spontaneous murine model of AE. These data further suggest common characteristics between the murine disease and human SpA and, in particular, PsA. 
Dactylitis is a characteristic feature of PsA, encountered in about one third of patients at some stage in the disease. ${ }^{3}$ It is more rarely recognised in other forms of SpA and seldom in sarcoidosis $^{9}$ and tuberculosis. ${ }^{10}$ In accordance with our findings in the reported animal model, recent magnetic resonance imaging and ultrasound studies have suggested that diffuse digital oedema and flexor tenosynovitis are the hallmark features of dactylitis. ${ }^{11}{ }^{12}$ McGonagle et al suggested that dactylitis may be caused by adjacent enthesitis. ${ }^{3}$ In the animals with dactylitis, however, no signs of synchronous AE or synovitis were seen. Similar data were recently reported in PsA using magnetic resonance imaging. ${ }^{11}$ Unlike other features of SpA, dactylitis may therefore not be linked to the enthesis. These new insights into dactylitis provide further evidence that PsA, in particular, cannot be explained by enthesitis alone.

$\mathrm{AE}$ with endochondral bone formation occurs rapidly in this animal model. Already within 4 weeks after clinical disease onset, not only extensive cartilage but also bone formation leading to joint space bridging is recognised. The number of joints affected steadily increases during the disease process. Eight weeks after clinical disease onset, most affected joints show signs of pathological bone formation, leading to joint space bridging and functional ankylosis. Although the mechanisms leading to $\mathrm{AE}$ are not clear, the possibility cannot be excluded that dactylitis triggers this process by the presence of an inflammatory reaction in proximity to the enthesis, as was suggested by our observations in two animals.

OPP has been described as a highly specific feature of PsA. ${ }^{56}$ Onychodystrophy, soft tissue swelling, and bone erosions of the distal phalanx typically characterise OPP. Our microscopy observations suggest that proliferative periostitis affects both the underlying bone and the overlying nail base in a continuous process. A similar mechanism may explain the strong relationship between distal interphalangeal joint arthritis/enthesitis and psoriatic nail involvement. Our observations are largely similar to the destructive inflammatory toe disease in different HLA transgenic mice lacking their own major histocompatibility complex. ${ }^{13}$

Remarkably, we did not see signs of skin involvement in DBA/l mice. On the other hand, stress factors have an important role in SpAD as in human psoriasis and PsA. Grouping of male mice, causing a substantial level of stress and aggressive behaviour, is required for the development of disease.

We therefore conclude that SpAD shows some unexpected but very striking similarities to PsA. Therefore SpAD is not only an attractive model for studying the process of AE, in particular of pathological cartilage and bone formation, but also for studying links between stress, inflammation, and pathological bone formation and for targeting specific genetic, metabolic, and inflammatory factors potentially relevant to PsA.

\section{ACKNOWLEDGEMENTS}

Rik Lories is the recipient of an "Aspirant" fellowship from the Fund for Scientific Research, Flanders. Patrick Matthys is the recipient of a post-doctoral fellowship from the Fund for Scientific Research, Flanders. This work was supported by research grant No G.0390.03 from the Fund for Scientific Research, Flanders.

\section{Authors' affiliations}

R J U Lories, K de Vlam, I Derese, F P Luyten, Laboratory for Skeletal Development and Joint Disorders, Department of Rheumatology, University Hospitals Leuven, Katholieke Universiteit Leuven, Belgium P Matthys, Laboratory for Immunobiology, Rega Institute, Katholieke Universiteit Leuven, Belgium

Correspondence to: Professor F P Luyten, Department of Rheumatology, University Hospitals Leuven, Herestraat 49, B-3000 Leuven, Belgium;

Frank.Luyten@uz.kuleuven.ac.be

Accepted 26 July 2003

\section{REFERENCES}

1 Gladman DD. Natural history of psoriatic arthritis. Baillieres Clin Rheumatol 1994:8:379-94.

2 Moll JM, Wright V. Psoriatic arthritis. Semin Arthritis Rheum 1973;3:55-78.

3 McGonagle D, Conaghan PG, Emery P. Psoriatic arthritis: a unified concept twenty years on. Arthritis Rheum 1999;42:1080-6.

4 Wright V. Seronegative polyarthritis: a unified concept. Arthritis Rheum 1978;21:619-33.

5 Goupille P, Laulan J, Vedere V, Kaplan G, Valat JP. Psoriatic onychoperiostitis. Report of three cases. Scand J Rheumatol 1995;24:53-4.

6 Boisseau-Garsaud AM, Beylot-Barry M, Doutre MS, Beylot C, Baran R. Psoriatic onycho-pachydermo-periostitis. A variant of psoriatic distal interphalangeal arthritis? Arch Dermatol 1996;132:176-80.

7 Corthay A, Hansson AS, Holmdahl R. T lymphocytes are not required for the spontaneous development of entheseal ossification leading to marginal ankylosis in the DBA/1 mouse. Arthritis Rheum 2000;43:844-51.

8 Nordling C, Karlsson-Parra A, Jansson L, Holmdahl R, Klareskog L. Characterization of a spontaneously occurring arthritis in male DBA/1 mice. Arthritis Rheum 1992;35:717-22.

9 Pitt P, Hamilton EB, Innes EH, Morley KD, Monk BE, Hughes GR. Sarcoid dactylitis. Ann Rheum Dis 1983;42:634-9.

10 Wessels G, Hesseling PB, Beyers N. Skeletal tuberculosis: dactylitis and involvement of the skull. Pediatr Radiol 1998;28:234-6.

11 Olivieri I, Salvarani C, Cantini F, Scarano E, Padula A, Niccoli L, et al. Fast spin echo-T2-weighted sequences with fat saturation in dactylitis of spondylarthritis. Arthritis Rheum 2002;46:2964-7.

12 Kane D, Greaney T, Bresnihan B, Gibney R, FitzGerald O. Ultrasonography in the diagnosis and management of psoriatic dactylitis. J Rheumatol 1999;26:1746-51.

13 Bardos T, Zhang J, Mikecz K, David CS, Glant TT. Mice lacking endogenous major histocompatibility complex class II develop arthritis resembling psoriatic arthritis at an advanced age. Arthritis Rheum 2002;46:2465-75. 